\title{
Artificial Pneumothorax Improves Radiofrequency Ablation of Pulmonary Metastases of Hepatocellular Carcinoma close to Mediastinum
}

\section{Taiyang Zuo}

Department of Interventional Oncology

Wenli Lin

Department of Interventional Oncology, Jinan Center Hospital

Fengyong Liu

Department of Interventional Radiology

Jinshun Xu ( $\nabla$ xujinshun@wchscu.cn )

Institution of Ultrasound Image \& Drug https://orcid.org/0000-0003-2420-2320

Research article

Keywords: lung metastasis, artificial pneumothorax, mediastinum, radiofrequency ablation, hepatocellular carcinoma

Posted Date: December 31st, 2020

DOI: https://doi.org/10.21203/rs.3.rs-53230/v2

License: (c) (i) This work is licensed under a Creative Commons Attribution 4.0 International License. Read Full License 


\section{Abstract}

Background: To investigate the feasibility, safety and efficacy of percutaneous radiofrequency ablation (RFA) of pulmonary metastases from hepatocellular carcinoma (HCC) contiguous with the mediastinum using the artificial pneumothorax technique.

Method: A total of 40 lesions in 32 patients with pulmonary metastases from HCC contiguous with the mediastinum accepted RFA treatment from August 2014 to May 2018 via the artificial pneumothorax technique. After ablation, clinical outcomes were followed up by contrast enhanced CT. Technical success, local tumor progression (LTP), intrapulmonary distant recurrence (IDR), and adverse events were evaluated. Overall survival (OS) and local tumor progression free survival (LTPFS) were recorded for each patient.

Results: The tumor size was $1.4 \pm 0.6 \mathrm{~cm}$ in diameter. RFA procedures were all successfully performed without intra-ablative complications. Technical success was noted in $100 \%$ of the patients. 5 cases of LTP and 8 cases of IDR occurred following the secondary RFA for treatment. Slight pain was reported in all patients. No major complications were observed. The 1, 2, and 3-year LTPFS rates were $90.6 \%, 81.2 \%$, and $71.8 \%$, and the 1,2 , and 3 -year OS rates were $100 \%, 100 \%$ and $87.5 \%$, respectively.

Conclusion: Artificial pneumothorax adjuvant RFA is a feasible, safe, and efficient method for treatment of pulmonary metastases from HCC contiguous with the mediastinum.

\section{Background}

Image-guided percutaneous local ablation has been widely used for the treatment of pulmonary metastasis, based on the high capability on accurate orientation of needles and real-time visualization of targets ${ }^{1-5}$. However, the minimal invasive thermal technique remains challenging for treatment of pulmonary metastasis abutting the mediastinum, as a result of the proximity to the heart or aorta. In 2007 , Iguchi et al reported that the primary technical effectiveness of radiofrequency ablation (RFA) was only $43 \%$ at 6 months postablation for treatment of 15 lung tumors contiguous with mediastinum ${ }^{6}$. Subsequently, studies on the efficacious improvement of RFA for treatment of lung tumors contiguous with mediastinum were rarely reported because of the insurmountable technique risk. Therefore, a safe and effective adjuvant method should be developed to reduce the risk of puncture and ablation for improvement of RFA efficiency of lung tumor contiguous with mediastinum.

Artificial pneumothorax is a standard technique that serves as an effective adjuvant for the clinical ablation of peripheral lung tumors 7,8 , which could be used for separation between the punctured needle path and normal tissues after gas administration into the pleural cavity ${ }^{9}$. It is suggested that the pulmonary tumors could be accurately separated from mediastinal structures during an artificial pneumothorax procedure. In that case, thermal damage to heart, aorta, or main nerves would be controlled, and pain caused by pleura heat could be effectively alleviated. In addition, the heat-sink effect 
of RFA could be reduced as well due to the artificial-induced space between tumors and vessels ${ }^{10}$. Therefore, this strategy could not only significantly reduce the risks of puncture and ablation, but also enhance RFA efficiency for complete ablation. Compared with the air administration to form artificial pneumothorax ${ }^{9}, \mathrm{CO}_{2}$ is less common but displays good properties including high diffusion coefficient, inert reaction to RFA, and pure gas injection without unidentified substance ${ }^{11}$. Therefore, we investigate the feasibility, safety and efficacy of using artificial $\mathrm{CO}_{2}$ pneumothorax in this study for mediating RFA of pulmonary metastases contiguous with the mediastinum.

\section{Methods}

\section{Patients enrollment}

This study was approved by the institutional ethics committee of our hospital, patients diagnosed with pulmonary metastases contiguous with the mediastinum who underwent RFA were retrospectively investigated at our hospital from August 2014 to May 2018. The target lesions of all cases were confirmed clinically or pathologically as hepatocellular carcinoma (HCC). "Contiguous with mediastinum" was defined as the distance between metastatic lesion and mediastinum was less than $3 \mathrm{~mm}$ on CT or MR images.

The inclusion criteria for this study were: I) a single metastatic lesion contiguous with mediastinum in the unilateral lobe of lung; II) lesion size less than $3 \mathrm{~cm}$ in diameter; III) orthotopic HCC shown to be inactive without recurrence and intrahepatic metastasis as indicated by enhance imaging after surgical and (or) interventional therapy; IV) no increase in numbers of pulmonary lesions after 1-2 months of observation; V) patients refuse to accept surgical resection; and VI) Karnofsky performance status (KPS) greater than $70 \%$.

The exclusion criteria were: I) patients with severe coagulation dysfunction including thrombocyte number $<30 \times 10^{9} / \mathrm{L}$, international normalized ratio (INR) $>3.0$, prothrombin time $>30 \mathrm{~s}$, or prothrombin activity (PTA) $<40 \%$; II) acute infection or chronic infection in acute phase; III) severe pulmonary hypertension defined as mean pulmonary artery pressure (mPAP) $>35 \mathrm{mmHg}$ measured by cardiac catheterization and (or) Doppler echocardiogram; IV) severe pulmonary insufficiency defined as $\mathrm{PaO}_{2}<$ $60 \mathrm{mmHg}$, with and without $\mathrm{PaCO}_{2}>50 \mathrm{mmHg}$; and $\mathrm{V}$ ) implantation of cardiac pacemaker.

\section{Artificial pneumothorax adjuvant RFA procedure}

All patients underwent contrast enhanced CT (CECT) of the chest prior to RFA in order to evaluate the anatomic relationship between lesions and peripheral cardiovascular structures. CECT was performed using the SOMATOM Force CT system (Siemens, Munich, Germany) with intravenous administration of Omnipaque (GE Medical, USA) as a contrast agent. Before the procedure, patients in a supine position received continuous electrocardiogram (ECG) monitoring and were hypodermically injected with $1 \%$ lidocaine. Then, the puncture point and needle track were determined by CT scan (MIYABI, Siemens, 
Munich, Germany). Subsequently, the biopsy needle was penetrated into the target in a direction away from the heart and vessels. When the procedure was accomplished, the biopsy needle was removed.

Thereafter, the artificial pneumothorax technique was performed using a 22-G puncture needle (NPAS100, COOK, USA) with a blunt tip. The needle was inserted along the well-designed puncture proposal, which specifies insertion route, depth and angle. When the needle tip reached the edge of the pleura, the needle core was pulled out, 1-2 ml of saline was injected locally to form a water capsule using T-stopcock and tubes. Then, saline in the tube flowed into the cavity, and the water capsule disappeared gradually as the needle tip continued to be inserted and reached into the pleura cavity. Then, $100-500 \mathrm{ml}$ of $\mathrm{CO}_{2}$ gas was frequently administered to separate the lung parenchyma. CT scanning was performed individually after injection of $100,200,300,400$, and $500 \mathrm{ml} \mathrm{CO}_{2}$ until the tumor was separated from the mediastinum and a feasible puncture path was established.

After the RF electrode was successfully inserted into the tumor based on the CT scanning assessment, percutaneous RFA (Model 1500, RITA Medical System, Mountain View, CA, USA) was performed at 60-70 $\mathrm{W}$ for $5-12 \mathrm{~min}$ at a rating temperature of $90^{\circ} \mathrm{C}$. During the process, an $18-\mathrm{G}$ unipolar electrode (Uniblate) was chosen (the length of the needle was $15 \mathrm{~cm}$ and the maximum ablation range of a single electrode was $3 \times 3 \mathrm{~cm}^{2}$ ). The ablation procedure was terminated when the ablation zone completely overlapped the target tumor and an ablative margin of $5-10 \mathrm{~mm}$ beyond the tumor boarder was achieved. Then, the RF electrode was withdrawn for coagulation of the needle track to avoid needle implantation and bleeding. During the procedure, 5-10 mg of morphine was intravenously injected for analgesia and sedation based on pain degree of patients. The ablation zone was defined as the pulmonary texture around the tumor that showed a circular exudation shadow with ground-glass appearance on CT imaging. After RFA, $\mathrm{CO}_{2}$ in the pleura cavity was aspirated with a 50-ml syringe and expelled through the T-stopcock until no additional gas could be aspirated.

\section{Follow up}

CECT was performed to evaluate the therapeutic response of tumors at $1,3,6$, and 12 months post ablation and at 6-month intervals thereafter. If incomplete tumor ablation was detected by CECT, secondary RFA was performed. According to the reporting criteria of image-guided tumor ablation 12,13, technical success was defined as a tumor that was treated according to the initial protocol and was covered completely by the ablation zone. Local tumor progression (LTP) was defined as the appearance of new tumor foci at the ablative margin. Intrapulmonary distant recurrence (IDR) was defined as any occurrence of a new tumor foci in the lung.

During follow-up, adverse events (AEs) after ablation, local tumor progression free survival (LTPFS), and overall survival (OS) were recorded for each patient. AEs were classified in this study according to $A E$ classification of the Society of Interventional Radiology ${ }^{14}$. The definition of major complication included moderate $A E$, severe $A E$, life-threatening or disabling event, patient death or unexpected pregnancy abortion. Mild AE was defined as minor complication. To assess the post-ablative pain, a visual analogue 
scale (VAS, a numerical rating scale: 0-10) was used based on previous publications: score 1-3 corresponding to slight pain, score 4-6 corresponding to moderate pain and score 7-10 corresponding to severe pain ${ }^{5,15}$.

\section{Statistical analysis}

Statistical analysis was performed using SPSS software (version 22.0; SPSS Inc, Chicago, IL, USA). Categorical variables were described as numbers (percentages). Continuous variables were described as mean \pm standard deviation (SD) or medians (range) according to the normality results using the Kolmogorov-Smirnov test. Survival was calculated by Kaplan-Meier survival analysis.

\section{Results}

\section{Patients characteristics}

The graphical diagram of this study is shown in Figure 1. A total of 32 patients with 40 pulmonary metastatic tumors contiguous with the mediastinum were confirmed from HCC using biopsy and then accept RFA treatment during this study. Prior to RFA treatment, no other treatment options were performed in all patients. As shown in Table 1, 62.5\% (20/32) of patients were males, and 37.5\% (12/32) of patients were females. The median age was 61 years old, ranging from 44 to 72 years old. The mean tumor diameter was $1.4 \pm 0.6 \mathrm{~cm}$ with a median distance of $0.1 \mathrm{~cm}$ from the mediastinum, ranging from 0 to $0.3 \mathrm{~cm} .87 .5 \%(28 / 32)$ patients had cirrhosis, mainly due to viral hepatitis B or C (96.4\%). In total, $56.2 \%$ (18/32) of patients were characterized as Child-Pugh classification A, and 43.7\% (14/32) of patients were characterized as Child-Pugh classification B. The median model for end-stage liver disease (MELD) score was 12 with range between 6 and 22.

\section{Therapeutic outcomes}

RFA technical success was achieved in all patients after the average $267.5 \pm 94.4 \mathrm{ml}$ administration of $\mathrm{CO}_{2}$ was performed in each case. One of the most representative cases was presented in Figure 2A-L. During the median follow-up of 29 months (ranging from 12 to 57 months), LTP occurred in 15.6\% (5/32) of patients and IDR occurred in 25\% (8/32) of patients (Table 2). Subsequently, the secondary RFA treatment were performed for the patients with LTP and IDR and technical success was obtained on CECT imaging assessment. The 1, 2, 3-year LTPFS rates after RFA were $90.6 \%, 81.2 \%$, and 71.8\%, and OS rates of 1,2 and 3 years were 100\%, 100\% and $87.5 \%$, respectively (Figure 3 ).

\section{Complications}

No major complications were observed during and following RFA procedure, such as severe pneumothorax, aeroembolism, hemothorax, lung abscess, alveolar bleeding, and tumor seeding. No AEs were relevant to the proximity of tumors to the mediastinum, such as vagus/recurrent laryngeal/phrenic nerve injury, vessel or oesophageal injury, pericardial effusion, arrhythmia, and cardiac infraction. The 
overall complication rate was $100 \%$ in the 32 patients with 40 tumors. All of them were minor complications including slight pain of VAS score 1-3 $(n=32)$, asymptomatic pneumothorax $(n=10)$, asymptomatic pleural effusion $(n=4)$, transient postablation syndrome of low-grade fever $(n=12)$ and general malaise $(n=16)$, as shown in Table 2. All complications resolved after 1-2 days postablation without any treatment.

\section{Discussion}

Artificial pneumothorax was first reported by Francini a century ago to treat pulmonary tuberculosis ${ }^{16}$. By injecting nitrogen between the parietal and visceral pleura, tuberculosis progression was suppressed by the reduction of blood flow and lymphatic reflux after pulmonary compression. During the past decades and enlightened by this report, artificial pneumothorax has been successfully performed for protection of chest wall ${ }^{17}$, relief of chest pain ${ }^{18}$, and biopsy of pulmonary or mediastinal tumors ${ }^{19}$.

In this study, the technique of artificial pneumothorax was first validated as a feasible, safe, and efficient adjuvant method for RFA upon treatment of pulmonary metastases contiguous with the mediastinum. As shown in the results, technique success of RFA was $100 \%$ (32/32) without major complications under artificial pneumothorax intervention, and the 1, 2, 3-year LTPFS rates were $90.6 \%$ and $81.2 \%$, and $71.8 \%$, respectively, resulting in the valid treatment efficiency and effective protection of proximate mediastinal structures. Compared to $43 \%$ only of the primary technical effectiveness reported by Iguchi et al, these results were comparable to the overall technique efficacy of standard RFA of lung intraparenchymal tumors away from mediastinum ${ }^{20,21}$. In general, our findings indicated that the use of artificial pneumothorax had safe and efficient local control for CT-guided RFA of lung metastasis contiguous with the mediastinum.

Given that $18-\mathrm{G}$ needles are commonly used for gas administration in thorax, a potential complication for that procedure is mainly iatrogenic pneumothorax $8,9,22,23$. To avoid damage to visceral pleura or pulmonary parenchyma, we used a 22-G blunt needle with negative pressure during the procedure and withdrew $\mathrm{CO}_{2}$ gas from the plural space after ablation. As a result, persistent pneumothorax or pleural hemorrhage with a chest tube drainage did not occurred.

In comparison with the air used before, the administration of $\mathrm{CO}_{2}$ to produce artificial pneumothorax in this study could be adsorbed more quickly by blood and reduce the risks of air embolism and injuries to normal tissues ${ }^{24}$. As a result, the blood oxygen saturations in all of the 32 patients were not influenced based on the ECG monitoring.

However, excessive $\mathrm{CO}_{2}$ in the pleural cavity can cause several adverse effects, including a) decrease in returned blood volume, increase in central venous pressure, and decrease in blood pressure; and b) impaired respiratory function and results in dyspnea, especially in the patients with poor pulmonary functions ${ }^{25}$. Furthermore, when excessive compression of lung parenchyma changes the electrical conductivity and heat conduction of RF, the ablation efficiency will be influenced, resulting in the damage 
enlargement of lung tissues ${ }^{9}$. Therefore, it is preferable to minimize the amount of $\mathrm{CO}_{2}$ injection during the process of artificial pneumothorax. Compared to the previously reported injection volume of 400-1400 $\mathrm{ml}^{22}$, the $100-500 \mathrm{ml}$ of injected $\mathrm{CO}_{2}$ in this study was more efficient to establish a safe puncture path between the tumor and mediastinum.

As known, resection is preferred for the treatment of pulmonary tumors, especially for the lesions contiguous with mediastinum. However, as to the patients with pulmonary metastasis, inoperability and intolerance are commonly coexisted because of old age, comorbidity, and operative suffering sentiment. Therefore, radiotherapy is the most common option for the treatment of pulmonary metastasis contiguous with mediastinum. Unfortunately, only $63 \%$ of 2-year OS rate was reported after 327 inoperable pulmonary oligometastases treated with stereotactic body radiotherapy (SBRT) ${ }^{26}$. Comparatively, Wah et al reported that the estimated OS rates at 1 and 3 years were $96.7 \%$ and $74.7 \%$, respectively, after 60 colorectal pulmonary oligometastases treated by RFA ${ }^{27}$. To the treatment of extrahepatic oligmetastases from HCC, Zhao et al have reported that the 1-, 2-year OS rates were $91 \%$ and $70 \%$, respectively ${ }^{28}$. Consistent with these results, 1-, 2-, 3-year OS rates of $100 \%, 100 \%$ and $87.5 \%$ were found in this study for the pulmonary oligometastases contiguous with mediastinum, attributing to the benefits of artificial pneumothorax adjuvant technique.

Of note, the technique is non-indicated in some cases, including patients suffering from pleural adhesion or severe respiratory insufficiency. Limitations on this study was related to the fact that only 32 patients with 40 tumors were subjected to this method, owning to the focus is on the metastatic tumors from HCC. Further studies should explore the artificial pneumothorax procedure as an adjuvant for RFA to understand the full extent of clinical benefits.

\section{Conclusion}

CT-guided RFA after artificially pneumothorax is a safe and effective method for the treatment of pulmonary metastases contiguous with the mediastinum. More samples should be examined to confirm the present results.

\section{Abbreviations}

RFA: radiofrequency ablation; HCC: hepatocellular carcinoma; KPS: Karnofsky performance status; INR: international normalized ratio; PTA: prothrombin activity; mPAP: mean pulmonary artery pressure; ECG: electrocardiogram; LTP: local tumor progression; IDR: intrapulmonary distant recurrence; AEs: adverse events; OS: overall survival; LTPFS: local tumor progression free survival; VAS: visual analogue scale.

\section{Declarations}

Ethics approval and consent to participate 
The study was approved by the Chinese PLA General Hospital Ethics Board. Due to the retrospective samples in the study, informed consent was waived.

\section{Availability of data and materials}

The datasets used and/or analyzed during the current study will be available from the corresponding author (xujinshun@wch.scu.cn) on reasonable request.

\section{Consent for publication}

Not applicable.

\section{Competing interests}

All authors declare no conflict of interest.

\section{Funding}

This work was supported by the National Natural Science Foundation of China (NSFC) (No. 81701797); Beijing Natural Science Foundation (No. 7192200); the 1.3.5 project for disciplines of excellence, West China Hospital, Sichuan University (ZYJC18008); Jinan science and technology project (No. 201805090); Science \& Technology Department of Sichuan Province (No. 2020JDRC0024); Chengdu Science \& Technology Bureau (2019-YF05-00376-SN); and Health Commission of Sichuan Province (20PJ011). All of the above funding was used to conduct data analyses and draft manuscript. None of the funding organizations were involved in the design of the study, implementation, management, and interpretation of the data.

\section{Author information}

Taiyang Zuo and Wenli Lin are co-first authors.

\section{Affiliations}

${ }^{1}$ Department of Interventional Oncology, Jinan Central Hospital Affiliated to Shandong University, Jinan, Shandong, 250013, China.

Taiyang Zuo, Wenli Lin

${ }^{2}$ Department of Interventional Radiology, Department of Interventional Ultrasound, Chinese PLA General Hospital, 100853, China.

Taiyang Zuo, Fengyong Liu, Jinshun Xu

${ }^{3}$ Department of Medical Ultrasound, Laboratory of Ultrasound Imaging Drug, West China Hospital, Sichuan University, Chengdu, Sichuan, 610041, China 
Jinshun Xu

\section{Author contributions}

Guarantors of integrity of entire study, all authors; study concepts and study design, JSX and TYZ; artificial pneumothorax adjuvant RFA procedure, TYZ and FYL; data collection, analysis and interpretation, all authors; manuscript drafting and revision, WLL, TYZ and JSX; literature research, WLL and JSX; statistical analysis, WLL; and manuscript final version approval, all authors.

\section{Corresponding authors}

Correspondence to Jinshun Xu.

\section{Acknowledgements}

Not applicable.

\section{References}

1. Smith $S L$, Jennings $P E$. Lung radiofrequency and microwave ablation: a review of indications, techniques and post-procedural imaging appearances. Br J Radiol 2015; 88(1046): 20140598.

2. de Baere T. Lung tumor radiofrequency ablation: where do we stand? Cardiovasc Intervent Radiol 2011; 34(2): 241-251.

3. Hiraki T, Gobara H, Fujiwara $\mathrm{H}$, et al. Lung cancer ablation: complications. Semin Intervent Radiol 2013; 30(2): 169-175.

4. Wei Z, Li Q, Ye X, et al. Microwave ablation or plus monochemotherapy in elderly advanced nonsmall-cell lung cancer patients. Minim Invasive Ther Allied Technol 2019: 1-9.

5. Xu J, Wu H, Han Z, et al. Microwave ablation of benign breast tumors: a prospective study with minimum 12 months follow-up. Int J Hyperthermia 2018; 35(1): 253-261.

6. Iguchi T, Hiraki T, Gobara $\mathrm{H}$, et al. Percutaneous radiofrequency ablation of lung tumors close to the heart or aorta: evaluation of safety and effectiveness. J Vasc Interv Radiol 2007; 18(6): 733-740.

7. Huang J, Hu Y, Mu X, et al. Thoracic ultrasound versus artificial pneumothorax in complications of medical thoracoscopy-a propensity score matching analysis. Journal of thoracic disease 2018; 10(9): 5269-5274.

8. Hou $X$, Zhuang $X$, Zhang H, et al. Artificial pneumothorax: a safe and simple method to relieve pain during microwave ablation of subpleural lung malignancy. Minim Invasive Ther Allied Technol 2017; 26(4): 220-226.

9. de Baere T, Dromain C, Lapeyre M, et al. Artificially induced pneumothorax for percutaneous transthoracic radiofrequency ablation of tumors in the hepatic dome: initial experience. Radiology 2005; 236(2): 666-670. 
10. Hocquelet A, Balageas P, Frulio N, et al. Aggressive Intrasegmental Recurrence of Periportal Hepatocellular Carcinoma after Radiofrequency Ablation: Role of Ablative Technique and Heat-Sink Effect? Radiology 2015; 276(3): 932-933.

11. Belteki G, Lin B, Morley CJ. Weight-correction of carbon dioxide diffusion coefficient (DCO2) reduces its inter-individual variability and improves its correlation with blood carbon dioxide levels in neonates receiving high-frequency oscillatory ventilation. Pediatr Pulmonol 2017; 52(10): 1316-1322.

12. Ahmed M. image-guided Tumor ablation: Standardization of Terminology and Reporting Criteria-A 10-Year Update. Radiology 2014; 273: 241-260.

13. Ahmed M. Image-guided tumor ablation: standardization of terminology and reporting criteria-a 10year update: supplement to the consensus document. J Vasc Interv Radiol 2014; 25(11): 1706-1708.

14. Khalilzadeh O, Baerlocher MO, Shyn PB, et al. Proposal of a New Adverse Event Classification by the Society of Interventional Radiology Standards of Practice Committee. J Vasc Interv Radiol 2017; 28(10): 1432-1437.e1433.

15. Deib G, Deldar B, Hui F, et al. Percutaneous Microwave Ablation and Cementoplasty: Clinical Utility in the Treatment of Painful Extraspinal Osseous Metastatic Disease and Myeloma. AJR Am J Roentgenol 2019: 1-8.

16. Garbarino MC, Cani V, Mazzarello P. A century ago: Carlo Forlanini and the first successful treatment of tuberculosis. Lancet 2018; 392(10146): 475.

17. Solomon SB, Thornton RH, Dupuy DE, et al. Protection of the mediastinum and chest wall with an artificial pneumothorax during lung ablations. J Vasc Interv Radiol 2008; 19(4): 610-615.

18. Hiraki T, Gobara $H$, Shibamoto $K$, et al. Technique for creation of artificial pneumothorax for pain relief during radiofrequency ablation of peripheral lung tumors: report of seven cases. $J$ Vasc Interv Radiol 2011; 22(4): 503-506.

19. Scalzetti EM. Protective pneumothorax for needle biopsy of mediastinum and pulmonary hilum. Journal of thoracic imaging 2005; 20(3): 214-219.

20. Jiang B, McClure MA, Chen T, et al. Efficacy and safety of thermal ablation of lung malignancies: $A$ Network meta-analysis. Annals of thoracic medicine 2018; 13(4): 243-250.

21. Hiyoshi Y, Miyamoto Y, Kiyozumi Y, et al. CT-guided percutaneous radiofrequency ablation for lung metastases from colorectal cancer. Int J Clin Oncol 2019; 24(3): 288-295.

22. Lin ZY, Li YG. Artificial pneumothorax with position adjustment for computed tomography-guided percutaneous core biopsy of mediastinum lesions. Ann Thorac Surg 2009; 87(3): 920-924.

23. Fujiwara $\mathrm{H}$, Arai $\mathrm{Y}$, Ishii $\mathrm{H}$, et al. Computed Tomography-guided Radiofrequency Ablation for Subdiaphragm Hepatocellular Carcinoma: Safety and Efficacy of Inducing an Artificial Pneumothorax. Acta Med Okayama 2016; 70(3): 189-195.

24. Favelier S, Guiu S, Cherblanc V, et al. Transthoracic adrenal biopsy procedure using artificial carbon dioxide pneumothorax as outpatient procedure. Cardiovasc Intervent Radiol 2013; 36(4): 1184-1187. 
25. Hill RC, Jones DR, Vance RA, et al. Selective lung ventilation during thoracoscopy: effects of insufflation on hemodynamics. Ann Thorac Surg 1996; 61(3): 945-948.

26. Shultz DB, Filippi AR, Thariat J, et al. Stereotactic ablative radiotherapy for pulmonary oligometastases and oligometastatic lung cancer. J Thorac Oncol 2014; 9(10): 1426-1433.

27. Zhong J, Palkhi E, Ng H, et al. Long-Term Outcomes in Percutaneous Radiofrequency Ablation for Histologically Proven Colorectal Lung Metastasis. Cardiovasc Intervent Radiol 2020; 43(12): 19001907.

28. Mu L, Sun L, Pan T, et al. Percutaneous CT-guided radiofrequency ablation for patients with extrahepatic oligometastases of hepatocellular carcinoma: long-term results. Int J Hyperthermia 2018; 34(1): 59-67.

\section{Tables}

Table 1. Characteristics of the 32 patients with $\mathbf{4 0}$ pulmonary metastatic tumors contiguous with the mediastinum. 


\begin{tabular}{ll}
\hline Characteristics & Values, $\mathbf{n}(\%)$ \\
\hline Age (y), median (range) & $61(44-72)$ \\
Sex & \\
$\quad$ Male & $20(62.5)$ \\
Female & $12(37.5)$ \\
Pulmonary metastatic tumors (cm) & \\
Tumor diameter (mean \pm SD) & $1.4 \pm 0.6$ \\
Distance from mediastinum, median (range) & $0.1(0-0.3)$ \\
Comorbidity & \\
Cirrhosis & $28(87.5)$ \\
Hypertension & $12(37.5)$ \\
Diabetes & $10(31.2)$ \\
Cardiovascular disease & $6(18.7)$ \\
Intrahepatic diseases & \\
Viral hepatitis B & $20(62.5)$ \\
Viral hepatitis C & $7(21.9)$ \\
Alcohol & $2(6.2)$ \\
Others & $3(9.4)$ \\
Child-Pugh class & \\
A & $18(56.2)$ \\
B & $14(43.7)$ \\
Laboratory data (mean \pm SD) & \\
AFP (ng/ml) & $88.7 \pm 67.2$ \\
Total bilirubin ( $19 m o l / 1)$ & $19.1 \pm 8.4$ \\
Albumin ( $/ \mathrm{l})$ & $35.2 \pm 6.7$ \\
Prothrombin time (\%) & $80.4 \pm 18.3$ \\
Platelet count (10^9/l) & $156 \pm 48$ \\
Creatinine (mmol/l) & $6.5 \pm 3.2$ \\
MELD score, median (range) & $12(6-22)$ \\
\hline
\end{tabular}

AFP: alpha fetal protein; MELD: model for end-stage liver disease (MELD); SD: standard deviation.

Table 2. RFA Outcomes of pulmonary metastases contiguous with the mediastinum $(n=32)$ 


\begin{tabular}{ll}
\hline & Values, n (\%) \\
\hline Follow up (months), median (range) & $29(12-57)$ \\
Technical success & $32(100)$ \\
Local tumor progression (LTP) & $5(15.6)$ \\
Intrapulmonary distant recurrence (IDR) & $8(25)$ \\
Major complications & 0 \\
Minor complications & \\
$\quad$ Slight pain & $32(100)$ \\
$\quad$ Asymptomatic pneumothorax & $10(31.2)$ \\
$\quad$ Asymptomatic pleural effusion & $4(12.5)$ \\
$\quad$ Low-grade fever & $12(37.5)$ \\
General malaise & $16(50)$ \\
\hline
\end{tabular}

\section{Figures}

\section{Inclusion}

1) a single metastatic lesion in the unilateral lobe

2) lesion size less than $3 \mathrm{~cm}$ in diameter

3) intrahepatic lesion in stability or inactivity 4) the number of pulmonary lesions does not increase after 1-2 months of observation

5) patients refuse to accept surgical resection

6) $\mathrm{KPS} \geq 70 \%$

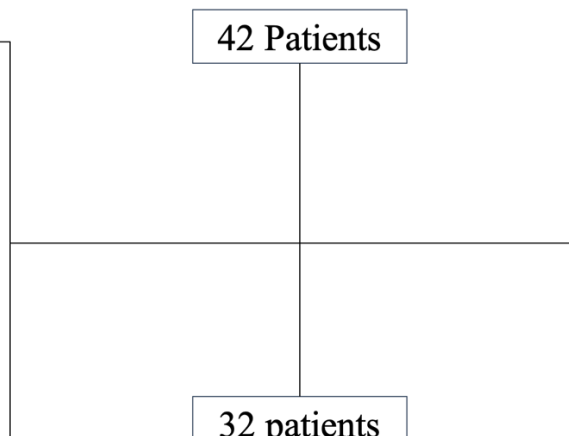

32 patients

\section{Exclusion}

1) severe coagulation dysfunction, $N=1$ 2) acute infection or chronic infection in acute phase, $\mathrm{N}=4$

3) severe pulmonary hypertension, $\mathrm{N}=1$

4) severe pulmonary insufficiency, $N=1$

5) implantation of cardiac pacemaker, $\mathrm{N}=3$

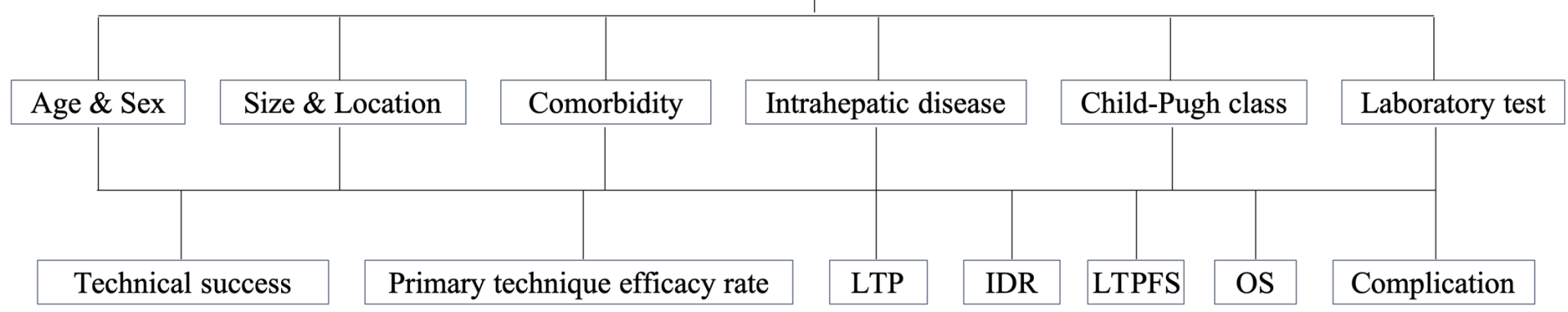

Figure 1

Graphical diagram of this study. KPS: Karnofsky performance status; LTP: local tumor progression; IDR: intrapulmonary distant recurrence; LTPFS: local tumor progression-free survival; OS: overall survival. 

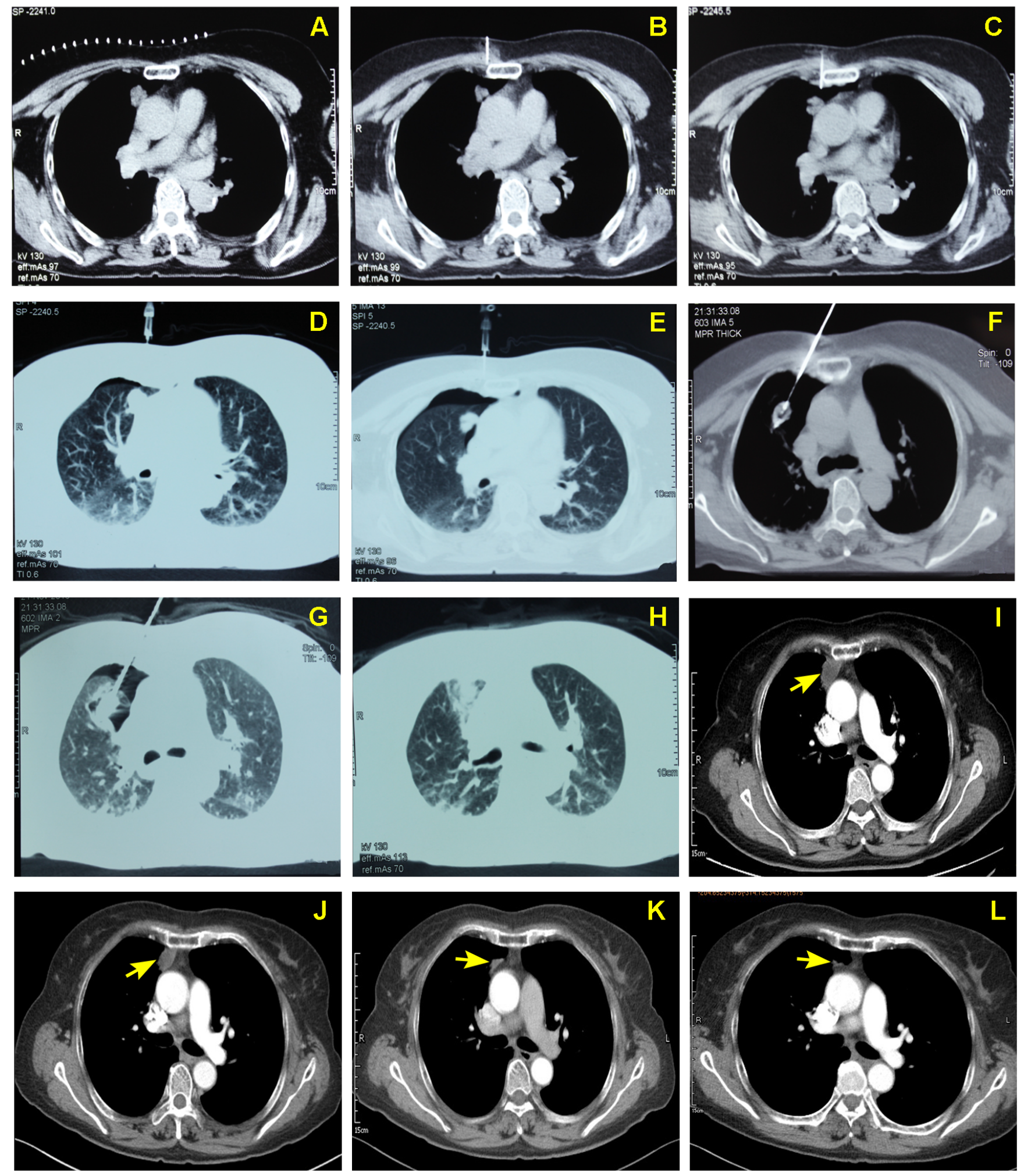

Figure 2

Artificial pneumothorax adjuvant RFA of pulmonary metastases contiguous to the mediastinum (a 67year-old man with a metastatic lesion in the superior lobe forepart of right lung). Tumor size, $1.5 \times 1.2 \mathrm{~cm}$. (A) Before ablation, chest CT imaging was performed to evaluate the anatomic relationship between tumor and peripheral cardiovascular structures. (B) Subsequently, a 22-G needle tip was used to create a puncture that reached the outer edge of pleura for injection of 1-2 $\mathrm{ml}$ saline. (C) The needle tip entered 
into the pleura, and the saline in the tube flowed into the cavity. (D-E) CO2 gas was administered gradually with a syringe until the tumor was separated from the mediastinum. (F) CT image during RFA showed the electrode inserted into the tumor and located away from the mediastinum by proxy of artificial pneumothorax. (G) The ablation zone gradually increased following the RFA procedure. $(H)$ After RFA, the pulmonary texture around tumor showed a circular exudation shadow with ground-glass appearance on CT image. (I) Contrast enhanced CT image 1 month after RFA showed no enhancement of the ablated tumor contiguous to the mediastinum. (J-L) The size of ablated tumor decreased gradually after RFA during follow up at 3, 6, and 12 months, respectively.
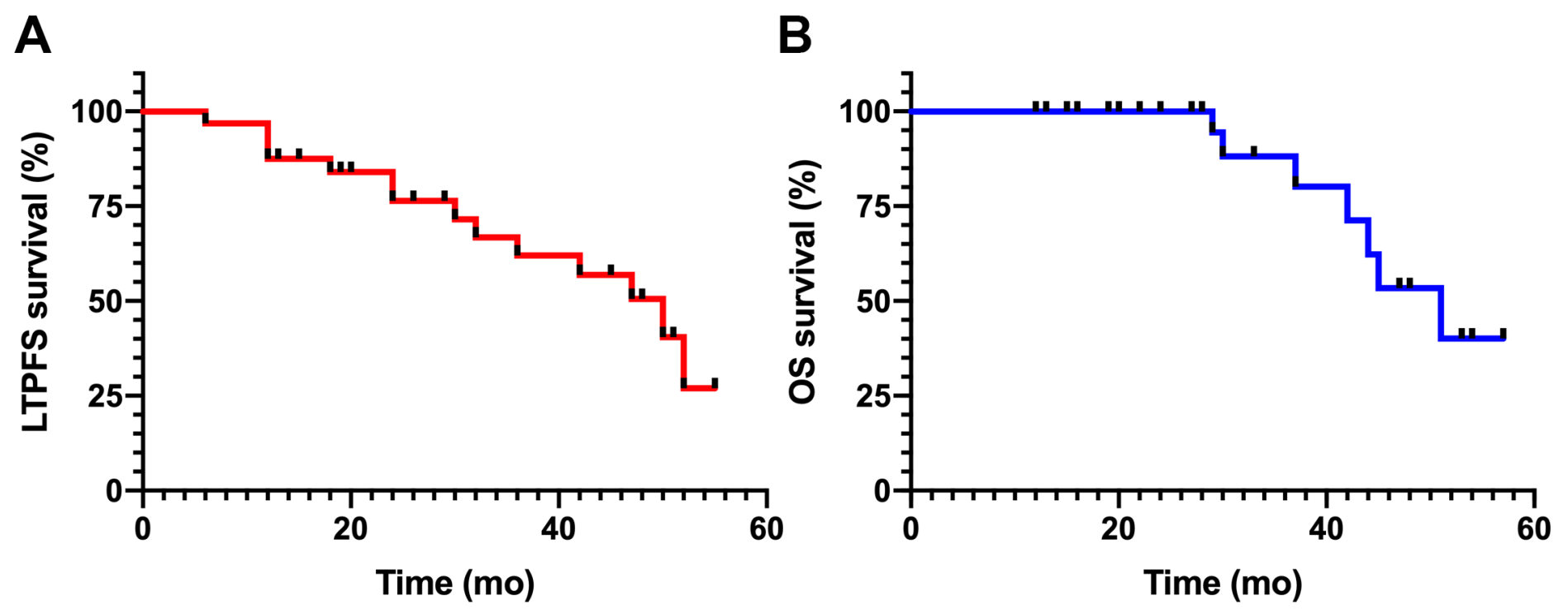

Figure 3

Overall survival (OS) and local tumor progression-free survival (LTPFS) curves during patient follow-up. 